Retos y experiencias en la construcción de la Paz.

\title{
Encuentros dialógicos para la prevención y resolución de conflictos: Una manera de mejorar las relaciones en la institución educativa Luis Carlos Galán Sarmiento de Itagüi, Colombia ${ }^{1}$
}

Dialogue meetings for the prevention and resolution of conflicts: a way to improve relationships in the educational institution Luis Carlos Galán Sarmiento from Itagüí, Colombia

Jorge Humberto Sánchez Franco Institución Educativa Luis Carlos Galán Sarmiento.

humbertosanchez2001@yahoo.com

\section{Resumen}

En el presente ensayo se describen algunas de las mejoras en la convivencia escolar obtenidas en la Institución Educativa Luis Carlos Galán Sarmiento del municipio de Itagüí, (Departamento de Antioquia - Colombia), resultado de la realización de los "Encuentros Dialógicos para la Prevención y Resolución de Conflictos", como una manera particular de implementar la Actuación Educativa de Éxito Modelo Dialógico de Prevención y Resolución de Conflictos, del Proyecto Comunidades de Aprendizaje.

\section{Abstract}

This essay describes some of the improvements in school obtained at the Luis Carlos Galán Sarmiento Educational Institution of the municipality of Itagüí, (Department of Antioquia Colombia); results of the realization of $<<$ Dialogical Meetings for the Prevention and Resolution of Conflicts $>>$ as a particular way to implement the successful educational actions Dialogic Model of Prevention and Resolution of Conflicts, of the Learning Communities Project.

Palabras claves: Convivencia, Prevención y Resolución de Conflictos, Comunidades de Aprendizaje, Diálogo Igualitario y Participación.

${ }^{1}$ Recibido: 19/04/2018 Evaluado: 23/05/2018 Aceptado: 25/08/2018 
Keywords: coexistence, conflict prevention and resolution, learning communities, egalitarian dialogue and participation.

\section{Contexto Social}

La Institución Educativa Luis Carlos Galán Sarmiento de Itagüí, departamento de Antioquia, se encuentra ubicada en los límites entre la zona urbana y rural. Contamos con 1.040 estudiantes en los niveles de educación preescolar, básica y media en tres jornadas: mañana, tarde y fines de semana. Los fines de semana atendemos educación de adultos orientada intencionalmente a la formación de las familias de los estudiantes de la educación regular. Nuestra población estudiantil en su gran mayoría hace parte de un contexto social caracterizado por la exclusión, como consecuencia de la violencia, los desplazamientos forzosos, las relaciones inequitativas de género, el microtráfico, la falta de oportunidades y el moldeamiento político. Este último se ve reflejado en la estratificación social, que para el caso en mención hace refiere a los estratos uno y dos que corresponden a los más bajos, y que popularmente son llamados de manera despectiva estrato cero, en tanto que, no gozan de los derechos a los servicios públicos mínimos, como acueducto y alcantarillado (como es el caso de la vereda La María). La planta física de la institución está ubicada justo en lo que el conflicto ha llevado a reconocer como fronteras invisibles. Se trata de una zona en litigio por el control del microtráfico entre bandas delincuenciales que han producido muerte, miedo y silencios que han afectado gravemente la convivencia dentro y fuera de nuestra escuela.

Las exclusiones sociales, la desigualdad de oportunidades y otras formas de violencia se instalaron en las aulas. El irrespeto por la palabra y por las ideas se había constituido en el común denominador, así como las agresiones cotidianas de todo tipo: físicas y simbólicas.

La lucha del maestro por hacerse escuchar, en un mundo absolutamente ruidoso y ensordecedor, terminaba siempre en la desesperanza. En la misma desesperanza que habitaba en todos los niños y adolescentes que venían a la escuela, no por gusto, sino obligados por sus padres para tratar de escapar de las calles y sus peligros. Las relaciones de los padres de familia con los maestros y directivos docentes, también estaban impregnadas de violencia. Relaciones tensas cargadas de exigencia de lado y lado que no permitían el establecimiento de acuerdos intencionados en el proceso de formación.

Sabíamos, todos sabíamos y anhelábamos otra forma de educación para salir del atolladero. ¿Cómo poder construir aprendizajes transformadores en un ambiente cargado de autoritarismos, de ausencia de sentido, de negación de la palabra propia y del otro, así como de actitudes violentas? Necesitábamos un mundo habitado por la palabra, por la escucha en igualdad de condiciones, que nos permitieran la transformación del acto educativo, para alcanzar ese anhelo de todos: otro tipo de educación que nos incluyera y nos liberara.

\section{Encuentro Transformador}

Una vez reconocida esta problemática social, la prioridad para nuestra Institución Educativa ha sido la construcción de una cultura de convivencia en el marco del aprendizaje dialógico 
mediante la implementación de la Actuación Educativa de Éxito Modelo Dialógico para la Prevención y Resolución de Conflictos. Entendemos el aprendizaje desde una perspectiva dialógica, caracterizada por un ejercicio democrático deliberativo, que posibilita el ejercicio de la solidaridad y el respeto en la construcción de sentido, que busca la transformación (Flecha, 1999).

En el Proyecto Educativo Institucional hemos adoptado la propuesta de Comunidades de Aprendizaje $^{2}$ como la estrategia de participación y filosofía de vida para alcanzar el sueño de una escuela y una sociedad en permanente transformación. Actualmente estamos desarrollando las siete Actuaciones Educativas de Éxito propuestas, orientadas al mejoramiento del aprendizaje, la convivencia y la disminución de las brechas de inequidad, mediante procesos de participación democrática y el establecimiento de una cultura de las interacciones en el marco del diálogo igualitario. De manera particular, estamos desarrollando en todos los grupos lo que hemos denominado $<<$ Encuentros Dialógicos para la Prevención y Resolución de Conflictos $>>$.

En el cuadernillo Modelo Dialógico de Resolución de Conflictos, adaptación hecha a partir del material de formación producido por CREA pág., 1, se define el Modelo de Prevención y Resolución de Conflictos como un modelo "basado en el diálogo como herramienta para superar las desigualdades. En este modelo la resolución del conflicto se da a través de un consenso entre todas las partes involucradas, especialmente los alumnos, sobre las normas de convivencia. Esas normas son elaboradas de forma colaborativa por toda la comunidad". Nuestro Encuentro Dialógico se fundamenta en la definición anterior, haciéndola realidad no sólo en los espacios semanales destinados para su desarrollo, sino mediante la apropiación intencionada de los siete principios del aprendizaje dialógico, como hilos conductores, en la implementación de todas las Actuaciones Educativas de Éxito y de manera especial, del principio del diálogo igualitario. Estos siete principios conducen a la formación de un aprendizaje dialógico que se produce "en diálogos que son igualitarios, en interacciones que se reconoce la inteligencia cultural en todas las personas y que están orientados a la transformación de los niveles previos de conocimiento y del contexto sociocultural para avanzar hacia el éxito de todos y todas. El aprendizaje dialógico se produce en interacciones que aumentan el aprendizaje instrumental, favorecen la creación de sentido, están guiadas por principios solidarios y en las que la igualdad de la diferencia son valores compatibles y mutuamente enriquecedores.

Hoy asistimos a lo que podríamos llamar otro mundo posible, una escuela que nos incluye, que nos forma y nos transforma mientras participamos de su transformación. Cada

\footnotetext{
${ }^{2}$ El Proyecto fue fruto de la Investigación INCLUD-ED realizada del 2005 al 2011 por el CREA de la Universidad de Barcelona. En Colombia, a partir del año 2014, Natura Cosméticos en alianza con la Fundación Empresarios por la Educación, las Secretarías de Educación y otros aliados empresariales, vienen acompañando su implementación en más de 100 escuelas de diferentes departamentos del país. Para más información ver: http://cort.as/-BkR8.
} 
vez nos escuchamos más y combatimos la violencia con actitudes de respeto y generación de oportunidades.

\section{Diálogo Igualitario, el camino}

Consideramos que el elemento transversal y sin el cual no sería posible alcanzar la intencionalidad del Modelo, es precisamente el Diálogo Igualitario. En el cuadernillo de Aprendizaje Dialógico, adaptación hecha a partir del material de formación producido por CREA, en su página 9 se define así: "El Diálogo igualitario ocurre siempre que se tienen en cuenta las contribuciones de todas las personas que participen en él. Todos han de tener la misma oportunidad de hablar y de ser escuchados, siendo que la fuerza está en la calidad de los argumentos, en el sentido de lo que se defiende, y no en la posición jerárquica de quien habla".

Un elemento fundamental de la definición del Aprendizaje Dialógico es el consenso. Para que éste sea posible, se hace necesaria una transformación cultural. No es posible el consenso en un sistema de participación piramidal caracterizado por la obediencia y la sumisión. Por eso, se requiere de la horizontalidad democrática emanada de la entraña liberadora que emerge de una participación responsable y con sentido, como la planteada por Comunidades de Aprendizaje.

Una horizontalidad democrática será posible en ambientes en los que el ejercicio de la autoridad sea un reconocimiento y no una imposición. Se habla de reconocimiento cuando la autoridad es compartida. Si la autoridad se distribuye, se incrementa. El compromiso personal y comunitario, es una consecuencia del ejercicio de la autoridad. En clave Freire (1970) podemos decir que actuar con el otro para transformar el mundo es una posibilidad de crecimiento personal e institucional.

Nuestras aulas se han venido convirtiendo en el laboratorio de la escucha con sentido. Estudiantes, maestros y padres de familia, comprometidos con el cambio, hemos hecho nuestro, el derecho deber de la palabra y la escucha, como condición posibilitadora del aprendizaje y la convivencia.

Gracias al diálogo igualitario hemos pasado del mundo de los ignorados al mundo de la existencia. Cuando somos escuchados, somos reconocidos. Esta es la gran fuerza de la transformación cultural que estamos viviendo.

\section{La participación, una experiencia preventiva y liberadora}

Rescatamos la participación de la comunidad en el desarrollo de la propuesta, como el eje fundamental que nos ha posibilitado el cambio gradual, de una estructura piramidal de la escuela, a una de horizontalidad democrática con sentido de pertenencia y creciente compromiso. Participación que se da a través de las Actuaciones Educativas de Éxito y mediante el ejercicio permanente de los siete principios del Aprendizaje Dialógico.

A continuación, entraremos a mencionarlos de manera muy general: 
Diálogo Igualitario: Se da en ambientes participativos en los que todos tienen el mismo derecho a hablar y ser escuchados, sus criterios se sustentan en la argumentación y no en la jerarquía de quien habla. Inteligencia Cultural: más allá de la inteligencia académica las interacciones humanas requieren de la inteligencia práctica y la inteligencia comunicativa, que brindan el contexto y hacen parte de la integralidad de las personas. Transformación: en nuestra propuesta el contexto y las personas se transforman mutuamente gracias a la participación. Mientras la educación tradicional es concebida para la adaptación social, nuestra propuesta liberadora busca la transformación, desde la transformación de las personas y sus interacciones. Ella se da gracias a la participación, en nuestro caso particular, mediante la planificación y el desarrollo del proyecto educativo institucional inspirado en los sueños de la comunidad educativa. Creación de Sentido: el aprendizaje es concebido para garantizar el crecimiento y mejora permanente de los estudiantes, generándoles sentido por lo que hacen, en tanto les garantiza su auto-realización personal mediante el desarrollo de su autonomía. Solidaridad: las actuaciones educativas de éxito son generadoras de un ejercicio de solidaridad, en tanto privilegian relaciones de equidad y justicia. Dimensión Instrumental: el desarrollo de competencias sociales, dialógicas y lógicas, así como la interiorización de contenidos y desarrollo de habilidades, están todas dirigidas a la inclusión social de las personas, para potenciarlas y así asegurar su papel en la sociedad. Igualdad de Diferencias: el verdadero sentido de democracia está en garantizarle a todos estar en el mismo punto de partida, representado en igualdad de oportunidades y expectativas de éxito, para que cada quien llegue donde quiera llegar, para alcanzar sus sueños sin diferencia de credo, raza, sexo, creencias y estrato social.

La Participación Educativa de la Comunidad involucra familiares, vecinos, docentes, directivos y personal administrativo, denominados voluntarios en la medida en que participan voluntariamente en la transformación de la escuela, mediante procesos de planificación, ejecución y acompañamiento. Participación que posibilita la generación de compromiso mediante la conformación de las Comisiones Mixtas para la toma de decisiones. Contamos con un número fluctuante de voluntarios entre 150 y 180 que participan en la consecución de los sueños institucionales e individuales, encaminados al mejoramiento del aprendizaje y la convivencia. Nuestro proceso de transformación democrática cuenta con las siguientes Comisiones Mixtas: Comisión Gestora, Comisión de Aprendizaje, Comisión de Sueños, Comisión de Recursos, Comisión de Voluntariado y Comisión de Convivencia.

Tanto las formas de participación, garantizadas en las Comisiones Mixtas, como en las Actuaciones Educativas de Éxito, han tenido como elemento articulador y generador de un ambiente seguro, el diálogo igualitario y las interacciones heterogéneas. En el Cuadernillo Modelo Dialógico de Resolución de Conflictos, adaptación hecha a partir del material de formación producido por CREA pág., 5, se establece que "Es necesario que los centros educativos sean espacios seguros para todos los niños y niñas, espacios en los cuales puedan aprender, crecer y relacionarse en libertad e igualdad". Hemos llegado a entender el modelo dialógico para la Prevención y Resolución del Conflicto como parte de un entramado cultural en el que cada día se incrementan los niveles de confianza gracias a la posibilidad de expresarse en lugares en los que existe el respeto por la palabra. Normalmente, no es fácil 
para un niño o niña, o para un adolescente expresar sus sentimientos y emociones ante un grupo. El miedo al ridículo o la burla, son muros en la comunicación que sólo es posible derrumbar mediante actitudes de mutuo respeto. Por lo tanto, el primer y principal acuerdo, en la implementación del modelo, es el respeto por el otro, el cual no puede ser el punto de partida o de llegada, sino el motor o el círculo virtuoso que posibilita las interacciones para el aprendizaje.

En cuanto a la prevención de las actitudes violentas, el desarrollo de los encuentros dialógicos para la prevención y resolución del conflicto promueve sanas relaciones de amistad, en tanto que privilegia el diálogo igualitario, puesto que la oportunidad de todos y todas de ser escuchados, les genera posibilidades de establecimiento de vínculos desde la valoración de las intervenciones propias y ajenas. En el cuadernillo Modelo Dialógico de Resolución de Conflictos, adaptación hecha a partir del material de formación producido por CREA pág., 14, se establece que "La socialización preventiva de la violencia de género consiste en motivar interacciones sociales que promuevan la atracción por los modelos igualitarios y el rechazo de los modelos violentos. Se trata, de potenciar la socialización que transmite la igualdad como algo atractivo y excitante".

Los progresos de los estudiantes han sido altamente significativos. A modo de ejemplo, su participación activa y con sentido en las tertulias literarias, aparte de la bondad comprensiva que alcanzan en la socialización de la interpretación de los textos leídos y su consecuente relación con la vida y con otros textos, destacamos como de singular importancia, el aprendizaje y puesta en escena del diálogo igualitario.

Cada día se incrementan los niveles de escucha, el respeto por la palabra en ambientes caracterizados por la argumentación y la estética del diálogo igualitario.

Gracias a estos espacios, la confianza en sí mismos y en los demás ha venido creciendo. La palabra de todos y todas vale, y el reconocimiento de las realidades de los demás facilita el reconocimiento de las realidades propias, generando con ello una esperanza creciente y posibilitadora.

\section{Algunas evidencias de nuestra metamorfosis convivencial}

Queremos resaltar como experiencia significativa de mejoramiento continuo, la creación de una cultura de convivencia, y su consecuente reconocimiento por el Instituto Colombiano de Normas Técnica y Certificación (ICONTEC), al concedernos la categoría Master en Convivencia en el año 2016. Dicha experiencia se viene dando gracias a la implementación de la Actuación Educativa de Éxito: Modelo Dialógico de Resolución de Conflictos. En todas las aulas de clase y a la misma hora, para garantizar que sea un espacio curricularizado, dos veces por semana los estudiantes se reúnen, los días lunes para hablar y reflexionar acerca de valores al interior del área de Ética, y los viernes para evaluarse y adquirir compromisos grupales e individuales tendientes a mejorar la convivencia, aplicando acciones preventivas y correctivas asociadas a los niveles de responsabilidad, admitidos por cada uno de los miembros del grupo de manera voluntaria. 
En este espacio denominado Encuentro Dialógico para la Prevención y Resolución de Conflictos los estudiantes elaboran de manera consensuada Pactos Pedagógicos. En las actas elaboradas por los mismos estudiantes quedan plasmados los compromisos que más adelante serán evaluados por ellos. Estas prácticas dialógicas han transformado de manera significativa nuestra manera de vivir y convivir. Son ellas una alternativa altamente eficiente para mejorar la convivencia, al punto que los procesos disciplinarios a los que estábamos acostumbrados, ahora son la excepción a la regla. Las anotaciones que hacíamos en los registros de seguimiento a comportamientos inadecuados están en vía de extinción. Hoy no tenemos estudiantes suspendidos y los traslados a otras instituciones se dan por asuntos familiares, ajenos a la institución, no por problemas de convivencia escolar.

Todo esto ha sido consecuencia de que tanto la prevención como la solución del conflicto, encontraron un "lugar” y un "cómo” desde la implementación del Modelo Dialógico.

Nuestros estudiantes han fortalecido sus niveles de autorregulación, llegando a ver el espacio del Encuentro Dialógico para la Prevención y Resolución de Conflictos como el más propicio para atender las situaciones que podrían afectar la convivencia. Pasando de las respuestas inadecuadas, impulsivas y agresivas, a momentos de reflexión y de diálogo, para encontrar soluciones consensuadas, mediadas por la puesta en escena de sus propios sentimientos, verbalizando sus emociones y reconociendo el alcance de la responsabilidad de sus actos.

Tanto estudiantes como profesores, víctimas de la educación conductista tradicional, y de la estructura jerárquica que imposibilita la participación, hoy podemos decir que Comunidades de Aprendizaje es la propuesta liberadora, gracias al diálogo igualitario y a la posibilidad de la participación.

Hoy sabemos que con Comunidades de Aprendizaje se mejora la calidad de los aprendizajes, se cierran las brechas de las desigualdades sociales, se mejoran las relaciones de género y se genera una cultura transformadora.

Transcribimos algunas apreciaciones de nuestros estudiantes a modo de ejemplo y para ilustrar la importancia significativa de los Encuentros Dialógicos para la Prevención y Resolución de Conflictos:

"Personalmente considero que este espacio para la prevención y resolución de conflictos aporta mucho al desarrollo de nosotros como personas, como jóvenes, debido a que aprendemos a ver el punto de vista de las otras personas, a respetarlo, a entender que podemos cambiar nuestros conceptos, nuestra mente. A saber que, así como yo tengo la razón, la otra persona también la tiene. A comprender la situación por la que está pasando la otra persona y a saber que nadie está por encima de nadie" (Estudiante grado 10).

"Gracias al diálogo nosotros hemos solucionado muchos problemas, hemos expresado lo que sentimos, mejoramos la escucha, la humildad, el respeto por nosotros mismos y por los compañeros" (Estudiante grado 8). 
El Encuentro Dialógico para la Prevención y Resolución de Conflictos, espacio también abierto a padres de familia, se ha constituido en el nervio y sustancia del mejoramiento de la convivencia institucional. Nuestra escuela es un recinto constructor de paz en tanto que la palabra, la escucha, la participación, la esperanza y el sentido de la existencia se han hecho posibles en la cotidianidad de las interacciones entre diferentes, con igualdad de oportunidades.

Entre los logros obtenidos más significativos está la participación de todos los miembros de la comunidad educativa en los procesos de transformación cultural. Los padres de familia ya no nos ven simplemente como el lugar para guardar a sus hijos, mientras van a trabajar, ahora mismo nos perciben como el lugar seguro y apropiado en el que pueden traer a sus hijos a continuar el proceso de formación, nos ven con nuevos ojos: somos aliados y constructores de sueños en el proceso formativo. La escuela liberadora nos unió.

Los estudiantes han pasado de ser recipientes de conocimiento a constructores de vida, a través del mejoramiento continuo y participativo en su proceso de aprendizaje. Y como consecuencia, los maestros otrora enseñantes, nos estamos transformando en apasionados aprendices.

Este cambio cultural nos ha propiciado mejorar la convivencia, incrementar aprendizajes y disminuir las brechas de inequidad, mejorando los resultados de pruebas externas e internas y disminuyendo la deserción escolar.

\section{Testimonios: Un encuentro... no una reunión más}

Para finalizar, me permito compartir una serie de testimonios que dan cuenta del proceso, transcritos por el profesor Fredy Rivera, docente del área Lengua Castellana y orientador de grupo.

No era fácil hablar del tema. La posibilidad de la llegada de la muerte a un estudiante es siempre un "tabú" para hablar con los demás. Es algo mucho más difícil de entender en las mentes de jóvenes, que muchas veces ven la vida, desde su perspectiva, con una especie de inmortalidad.

Yessid. Un joven con problemas cardíacos. Sobreviviente de una operación que determinó el ritmo de su corazón con la ayuda de un marcapasos. Crecimiento en los huesos de forma desmedida, daño en su torax por el mismo problema. Tranquilo. Relajado. Amante del rock. Con una madre que en su desesperación, a veces más confundida que él, preocupada porque cada aliento de su hijo se prolongue. Guerrera siempre al lado de su "pequeño" de $1.91 \mathrm{mt}$.

Todos en el grupo entienden bien la situación de Yessid. Ese compañero que falta frecuentemente a clase, pero que cuando va a estudiar todos lo miran con afecto entendiendo a veces que sea la última vez que lo tienen en el salón.

Cada día que falta preguntan por él.

En los encuentros dialógicos de prevención y resolución de conflictos este muchacho de 16 años expresaba con frialdad cómo a veces, ver el mundo de forma optimista no valía la pena. Tal vez tantas enfermedades no le permitían ver sus valores. 
Es así como ante cuestionamientos por su autodestrucción, sus respuestas se manifestaban en comportamientos como fumar, beber licor, salir hasta altas horas de la noche. Todo como una forma de autodestrucción $u$ otra forma de vida, para encontrarle sentido a su existencia.

Vivir lo que no se ha vivido era casi siempre el mensaje de sus intervenciones. Muchas veces el grupo lo alentaba ya fuera en aula o por fuera de ella.

Una operación vendría a inquietarlo de nuevo. Como es normal los nervios afloran en todo su entorno. Y esta nueva experiencia tuvo impacto en los estudiantes del grupo 9B. Muchos temían al desenlace fatal. Otros aún con optimismo ni se atrevían a hablar sobre el tema.

Mientras tanto, Yessid muy deprimido, aterrado por su futuro incierto, no vendría a estudiar por algún tiempo.

Aparece de nuevo y las preguntas no cesan sobre su estado en las tertulias, las clases en general, y los encuentros dialógicos. Pero esta vez no eran los mismos comentarios deprimidos. Yessid vino con una visión más optimista. Comentaba sobre la muerte como una posibilidad, pero no como un futuro marcado. Hablaba de su experiencia desde una visión positiva. Comentaba cómo había ayudado a otros para mirar la vida desde una mejor perspectiva. De la forma cómo se ha sentido apoyado por sus compañeros. Cómo ha valorado cada vez que preguntan por él. Cada llamada, nota o comentario, cada voz de aliento, cada saludo y sonrisa. Es difícil escuchar esos comentarios sin que se toquen las fibras del alma y muchos estudiantes dejaban ver sus emociones con sollozos tímidos o con silencios que daban cuenta de la reflexión. Los muchachos del grupo no creían que las acciones, comentarios, frases de ayuda a su compañero, casi normales para ellos, lograrían cambiar la visión del mundo de alguien marcado para vivir al lado de intervenciones quirúrgicas, médicos y hospitales. Yessid hablaba y el asombro de los demás llegaba hasta las lágrimas de algunos que interpretaban esas oraciones como una despedida; mensajes que quedarían en sus mentes. Una parábola que justificara la vida misma.

Hoy sobrevive otra operación. A su casa han llegado muchos saludos, visitas y notas de aliento. Feliz, recibe cada mensaje. Encuentra en sus compañeros una razón más para vivir.

Todos han aprendido que la visión del mundo puede cambiar. Que vale la pena luchar por los ideales, independientemente de las situaciones adversas que estén viviendo. Yessid ha entendido con la ayuda de sus compañeros que no está solo. Que no lo estará mientras haya amigos que lo acompañen y lo entiendan.

Nada de esto hubiera sido posible si no se hubieran escuchado, si el encuentro dialógico no se hubiera facilitado. Desnudar su alma ante los demás no es nada fácil y menos en un grupo donde hay tantos desconocidos. Pero todos hemos aprendido el valor de escucharnos más allá de los comentarios comunes de un aula de clase. Más allá de llamados al orden y la disciplina, de las estructuras comunes, de formalismos. El diálogo entre los estudiantes les ha posibilitado el encuentro, dándoles más puntos en común que los que los dividen. Son diferentes, sí. Pero juntos en nuevas experiencias, en el viaje de la vida. (Fredy Rivera, docente Lengua Castellana, grados 8 y 9$)$. 


\section{Referencias}

CREA. (2011). Proyecto INCLUD-ED. Strategies for inclusion and social cohesion in Europe from Education. Extraído de http://cort.as/-A5go

Freire, P. (1970). Pedagogía del oprimido. Madrid:siglo XXI.

Flecha, R. (1999). Trabajar en la diversidad, superar la desigualdad. Aprendizaje dialógico en la sociedad de la información. En Actas del XVIII Encuentro Estatal de la Confederación de MRPs. Extraído: http://cort.as/-A5h0

INCLUD-ED. (2011). Actuaciones de éxito en las escuelas europeas. En Estdudios Creade No 9. Madrid: Secretaría General Técnica. Ministerio de Educación, IFIIE. Extraído de http://cort.as/-A5hn

¿Sabes lo que es una Comunidad Aprendizaje? (sf). Instituto Natura. Extraído de http://cort.as/-A5gc 